\title{
MPB64 DNA vaccine with immunogenicity and efficacy against tuberculosis
}

\author{
Yunyi Yao ${ }^{1}$, Qian $\mathrm{Hou}^{2}$, Hua $\mathrm{Jin}^{3}$, San Lin ${ }^{4}$, Yajun Wang ${ }^{5}$, Jianhong Xue ${ }^{6}$, Li Sun ${ }^{1}$, \\ Huarong Hong ${ }^{7}$, Xiaoli Zhou ${ }^{8}$ and Fang Chen ${ }^{1 *}$ \\ ${ }^{1}$ School of Life Sciences, Sichuan University, Chengdu, Sichuan, 610064, P.R. China. \\ ${ }^{2}$ The No.148 Hospital of PLA, Zibo, Shandong, 255300, P.R. China. \\ ${ }^{3} 2^{\text {nd }}$ Department of Gynecological Oncology, Affiliated Tumor Hospital, Xinjiang Medical University, Urumqi, Xinjiang, \\ 830011, P.R. China. \\ ${ }^{4}$ Departments of Thyroid Surgery, The First Affiliated Hospital of Jilin University, Changchun, Jilin, 130021, P.R. China. \\ ${ }^{5}$ College of Horticulture, Jinlin Agriculture University, ChangChun, Jilin, 130118, P. R. China. \\ ${ }^{6}$ International medical center, Chinese PLA General Hospital, Beijing 100853, P.R. China. \\ ${ }^{7}$ School of Basic Medical Science, Chengdu Medical College, Chengdu, Sichuan, 610083, P.R. China. \\ ${ }^{8}$ Sichuan Provincial School of Health, Chengdu, Sichuan, 610100, P.R. China.
}

Accepted 24 January, 2012

\begin{abstract}
Tuberculosis is a serious infectious disease caused by Mycobacterium tuberculosis. DNA vaccination is an advanced technique for protecting human bodies from infectious diseases including tuberculosis by injecting exogenous gene engineering DNA into the body to produce an immunological response. In this study, we examined the immunogenicity and protective efficacy of DNA vaccine (pCDNA-MPB64) expressing MPB64 protein and its booster effects in mice for controlling tuberculosis. The results showed that MPB64 DNA vaccine led to a dramatic augmentation of humoral and cellular responses. All these suggested that MPB64 DNA vaccines is an ideal vaccine and may be further developed as a useful method to prevent tuberculosis.
\end{abstract}

Key words: Tuberculosis, DNA vaccines, MPB64, recombination plasmid, antibody titer.

\section{INTRODUCTION}

Tuberculosis is a common, serious and sometimes lethal infectious disease caused by Mycobacterium tuberculosis. Tuberculosis usually attacks the lungs, while sometimes it also attacks other organs of human body such as bone, ovary and stomach (Sala et al., 2011). The main distribution of tuberculosis is located in many Asian and African countries. The most common vaccine against tuberculosis currently is Bacillus Calmette-Guérin (BCG), prepared from a strain of the attenuated or weakened live bovine tuberculosis bacillus. However, the protection effect of BCG sometimes is not certain, sometimes the immune results are not well as expected (Rouanet et al., 2010). What is more, BCG could interfer the test of

\footnotetext{
*Corresponding author. E-mail: fangchen151@yahoo.com.cn.
}

purified protein derivative (PPD, also known as the Mantoux screening test), a diagnostic tool for tuberculosis, making the PPD test very confused (Kernodle et al., 2010). Moreover, it makes the routine quarantine more difficult after vaccinated with BCG since natural infection and artificial immunity would be undistinguished. And the safety of BCG is still need to be improved. So it is very important to discover the new vaccine for the diagnosis and prevention of the tuberculosis.

DNA vaccines are one of the ideal vaccines. They are third generation vaccines, made up of a plasmid containing exogenous genetically engineered DNA in order to produce antigens (Kaufmann et al., 2011; Martín et al., 2011). DNA vaccines elicit a quick and ideal immunological response when highly active expression vectors used and thus result in an ideal protection for human bodies (Hawkridge et al., 2011). No risk for 
infection, long-term persistence of immunogen and focused immune response are the advantages of new DNA vaccines compared with the other traditional vaccines (Frantz et al., 2011; Okada et al., 2011; Liang et al., 2011). What's more, ease of development, production, storage and shipping, together with cost-effectiveness, are also the advantages of new DNA vaccines (Hanif et al., 2010). Thus, it is important to design a new DNA vaccine to better prevent tuberculosis. This is the main task and purpose of this research.

\section{MATERIALS AND METHODS}

\section{Construction of recombinant vector pCDNA-MPB64}

Genes coding MPB64 was amplified by PCR with primers and the genomic DNA of $M$. tuberculosis H37Rv as the template. The PCR products were first digested with $B a m H I$ and $E c o R I$ and then cloned into the corresponding sites eukaryotic expression vector pCDNA, resulting in recombinant plasmids named pCDNA-MPB64, respectively. The correctness of recombinant plasmids was confirmed by DNA sequencing and enzyme digestion. Plasmids pCDNA-MPB64 for DNA immunization were transformed into competent Escherichia coli DH5a, and endotoxin-free plasmid DNA was purified using the Qiagen Plasmid Giga kit (Qiagen, Valencia, $\mathrm{CA}$ ) according to the manufacturer's instructions. The purity of the recombinant vector was detected by ultraviolet spectrophotometer and evaluated according to OD260/OD280.

\section{Transfection of BHK-21 Cells and detection}

BHK-21(ATCC) cells were transfected with pCDNA-MPB64 and pCDNA by Lipofectamine 2000 (Invitrogen, USA). Cells at 90 to $95 \%$ confluence were transfected with $5 \mu \mathrm{g}$ vectors. After $6 \mathrm{~h}$ exposure, the normal culture medium, RPMI 1640 supplanted with $15 \%$ horse serum (Gibco, USA) was added into cells for another 18h-42h. RTPCR and Western blot were used to detect the effect of transfection. $\begin{array}{lcrrr}\text { Oligonucleotide primers of } & \text { MPB64 } & \text { (Up } & 5^{\prime} \\ \text { TTGCTCTGTTGTTCGGGTGTCGC } & 3^{\prime} & & \text { Down }\end{array}$ 5'AAGGCCTTGTACGTGGTCGTTG 3') were synthesized by Takara Company. After reverse transcription, cDNA was used as the templates for PCR (PE, 9600) and PCR product was detected for the gene expression. Total protein extracted $(15 \mu \mathrm{g})$ from the cells was boiled at $100^{\circ} \mathrm{C}$ with loading buffer for $5 \mathrm{~min}$, and then subjected to $12.5 \%$ SDS-PAGE. After electrophoresis, transfer was performed at $70 \mathrm{~V} 2 \mathrm{~h}$ at $4^{\circ} \mathrm{C}$. After blocking in $5 \%$ Nonfat milk for 1 $\mathrm{h}$, membranes were incubated overnight at $4^{\circ} \mathrm{C}$ with primary antibody (rabbit polyclonal IgG, Millipore) and secondary antibody (Goat Anti-Rabbit IgG, QIAGEN) for $1 \mathrm{~h}$ at room temperature in blocking solution. Photos were taken with film exposures. Betaactin was used as control gene both in RT-PCR and Western blot.

\section{Animal immunization}

Specific pathogen-free (SPF) 6- 8- week-old, male C57BL/6 mice (Lab Animal, Chengdu, China) were bred in cages on the animal feeding cabinet in a bio-safety level 3 laboratory. Mice received free access to food and water throughout the study. The research protocol was reviewed and approved by Sichuan University Committees on Bio-safety and Animal Care and Use Committee of China. Mice were randomly divided into (20 mice in each group): non-vaccinated control, vector control pCDNA, pCDNA-MPB64, BCG. Pasmid DNA (100 $\mu \mathrm{g})$ was injected intramuscularly in the same area, and immunization was repeated thrice with 2-week intervals.

\section{Detection of antibody titer in serum in immunized mice}

Blood were collected 3 weeks after immunization from mice tail. Serum were separated and stored at $-20^{\circ} \mathrm{C}$. ELISA was used to detect the antibody titer in serum. MPB64 was diluted to a final concentration of $10 \mu \mathrm{g} / \mathrm{ml}$ in coating buffer. $100 \mu \mathrm{l}$ solution were transfered to each well of a high affinity, protein-binding ELISA plate and incubated at $4^{\circ} \mathrm{C}$ overnight. Solution was flicked off and washed $3 \mathrm{~min} /$ time $\times 3$ times with PBST, and blocked using $100 \mu \mathrm{l}$ of $1 \% \mathrm{BSA}$ in each well and incubated at $37^{\circ} \mathrm{C}$ for $1 \mathrm{~h}$. Solution was flicked off and washed $3 \mathrm{~min} /$ time $\times 3$ times with TPBS, Serum were diluted to desired concentrations(1:50, 1:100, 1:150, 1:200, $1: 250 \ldots)$ in Blocking Solution and $100 \mu \mathrm{l}$ were added per well to the ELISA plate and incubated at room temperature for $2 \mathrm{~h}$. Solution was flicked off and washed $3 \mathrm{~min} / \mathrm{time} \times 3$ times with TPBS. The HRP-labeled rabbit anti mouse lgG was diluted (1:500, Invitrogen, USA) in Blocking Solution. $100 \mu \mathrm{l}$ of diluted antibody were added to each well and incubated at $37^{\circ} \mathrm{C}$ for $2 \mathrm{~h}$. Solution was flicked off and washed $3 \mathrm{~min} / \mathrm{time} \times 3$ times with PBS/Tween. $100 \mu \mathrm{l}$ of substrate solution was added and incubated at room temperature for $30 \mathrm{~min}$ avoiding light. $50 \mu \mathrm{l}$ of suspending solution were added and the optical density (OD) for each well were recorded with a microplate reader (Ray Biotech, Inc) set $\lambda$ to $492 \mathrm{~nm}$.

\section{Detection of IL-2 in immunizated mice}

To detect IL-2 in serum of mice after immune vaccine, ELISA was conducted by using assay kit. The mice IL-2 enzyme-linked immunosorbent assay (ELISA) kit (Promega, USA) is an in vitro ELISA for the quantitative measurement of rats IL-2 in serum, plasma, cell culture supernatants and urine. This assay employs an antibody specific for rats IL-2 coated on a 96-well plate. Standards and samples are pipetted into the wells and mice IL-2 present in a sample is bound to the wells by the immobilized antibody. The wells are washed, and then biotinylated anti-mice IL-2 antibody was added. After washing away unbound biotinylated antibody, HRPconjugated streptavidin is pipetted to the wells. The wells are again washed, a TMB substrate solution is added to the wells and color develops in proportion to the amount of rats IL-2 bound. The stop solution changes the color from blue to yellow, and the intensity of the color is measured at $450 \mathrm{~nm}$ (Ray Biotech, Inc).

\section{Statistical analysis}

All values were expressed as mean \pm SD. The results were evaluated by one-way ANOVA and Tukey's multiple comparison tests. Statistically significant differences between groups were defined as $p<0.05$. Calculations were performed with SPSS 12.0 software (SPSS, Chicago, USA).

\section{RESULTS}

\section{Construction and identification of expressing plasmid}

The gene of MPB64 was first amplified by PCR and $M$. tuberculosis H37Rv genomic DNA as the template. The purified PCR productions were digested with $\mathrm{BamHI}$ and 


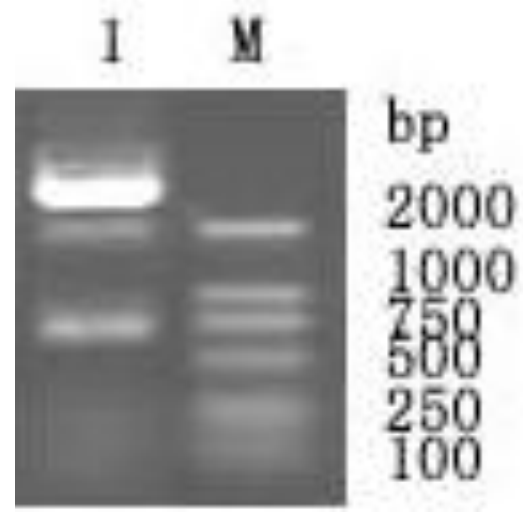

Figure 1. Construction of recombinant plasmids. Line 1: Product of pCDNA-MPB64 digested by enzyme; M:DNA Marker/DL2000.

EcoRI and separately cloned into the plasmid and pCDNA predigested with the same restriction enzymes, in order to construct eukaryotic expression plasmid pCDNA-MPB64. The positive recombination expressing plasmids were confirmed by analysis of the base pairs of plasmid, enzyme digest and PCR amplication. The pCDNA linear section and 620 bp insert section were generated after digestion of recombination expressing plasmids with BamH I and EcoR I (Figure 1). The same base pairs of DNA were generated after PCR amplication. All these indicated that the recombination expressing plasmid pCDNA-MPB64, which could translate MPB64 protein, was constructed successfully.

\section{Result of detection for BHK-21 cells transfected with vectors}

As shown in Figure 2, the gene expression of MPB64 was detected by RT-PCR. Positive result could be detected in BHK-21 translated with recombination expressing plasmid pCDNA-MPB64, while negative result could be detected in BHK-21 translated with pCDNA as control vector. As shown in Figure 3 , the protein expression of MPB64 was detected by Western blot. Positive result could be detected in BHK-21 translated with recombination expressing plasmid pCDNA-MPB64, while negative result could be detected in BHK-21 translated with pCDNA as control vector. This means the MPB64 was expressed in BHK-21 translated with recombination expressing plasmid pCDNA-MPB64.

\section{Result of antibody titer detection in mice serum}

As shown in Figure 4, the antibody titer in the serum was obviously increased significantly with the increased immune days. The antibody titer in the serum was obviously increased significantly in compare with control group, and pCDNA-MPB64 group and BCG group had no significantly difference, which showed that MPB64 vaccine could induce strong humoral immunity in vaccinated mice.

\section{Expression of IL-2 in serum.}

To determine cell-mediated immune responses in the serum, imunized mice were prepared 2 weeks after the last DNA vaccination. The expression levels of IL-2 in serum of vaccinated mice were determined by ELISA. As shown in Figure 5, IL-2 response increased in all groups except the vector control group. Mice vaccinated with $B C G$ induced the highest levels of IL-2 responses in the serum. Moreover, MPB64 group were significantly higher than control group $(P<0.05)$, and MPB64 group and BCG group had no significantly difference. These results showed that MPB64 could cause cell-mediated immune responses.

\section{DISCUSSION}

Tuberculosis (TB) is a common and sometimes lethal infectious disease caused by various strains of Mycobacterium tuberculosis (MTB). The main pathogenic bacteria of TB, MTB, are a small aerobic non-motile bacillus $\left(\mathrm{G}^{+}\right)$. MTB could withstand weak disinfectants and survive in a dry state for several weeks (Sarkar et al., 2011). Tuberculosis usually attacks the lungs through the air but can also affect other body organs such as bone, stomach and ovary. Most infections in humans are asymptomatic and latent, however, without proper treatment, some infected person would be dead finally. Strains of tuberculosis with antibiotic resistance, sometimes even with multi-drug-resistant are growing. Prevention of TB relies on screening programs, early detection and vaccination, among which Bacillus Calmette-Guérin vaccine (BCG) is most often used (Amaral et al., 2011).

Vaccines are one of the greatest achievements of modern medicine. It is one of the most effective methods to prevent zymosis including tuberculosis. DNA vaccination is a new technique for protecting the human body from epidemic diseases by injecting genetically engineered DNA, also called foreign DNA, into bodies to produce immunological response. To get the best immune response, highly active expression vectors are required (Mkrtichyan et al., 2008). These expression plasmids usually consist of a strong promoter to drive the in vivo transcription and translation of the target gene. They could elicit and stimulate the body to produce certain antibody to protect itself. These new vaccines have a number of advantages over conventional vaccines, including the ability to induce a wider range of immune response types, no risk for infection, long-term persistence of immunogen and focused immune 
Left: pCDNA Right: pCDNA-MPB64

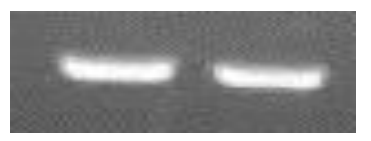

beta-actin

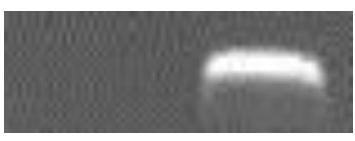

MPB64

Figure 2. Gene assay for BHK-21 cell transfected with pCDNA and pCDNA-MPB64.

\section{Left: pCDNA Right: pCDNA-MPB64}

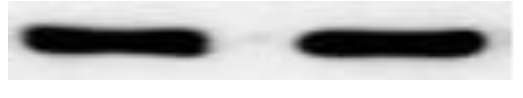

beta-actin

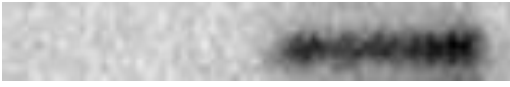

MPB64

Figure 3. Protein assay for BHK-21 cell transfected with pCDNA and pCDNA-MPB64.

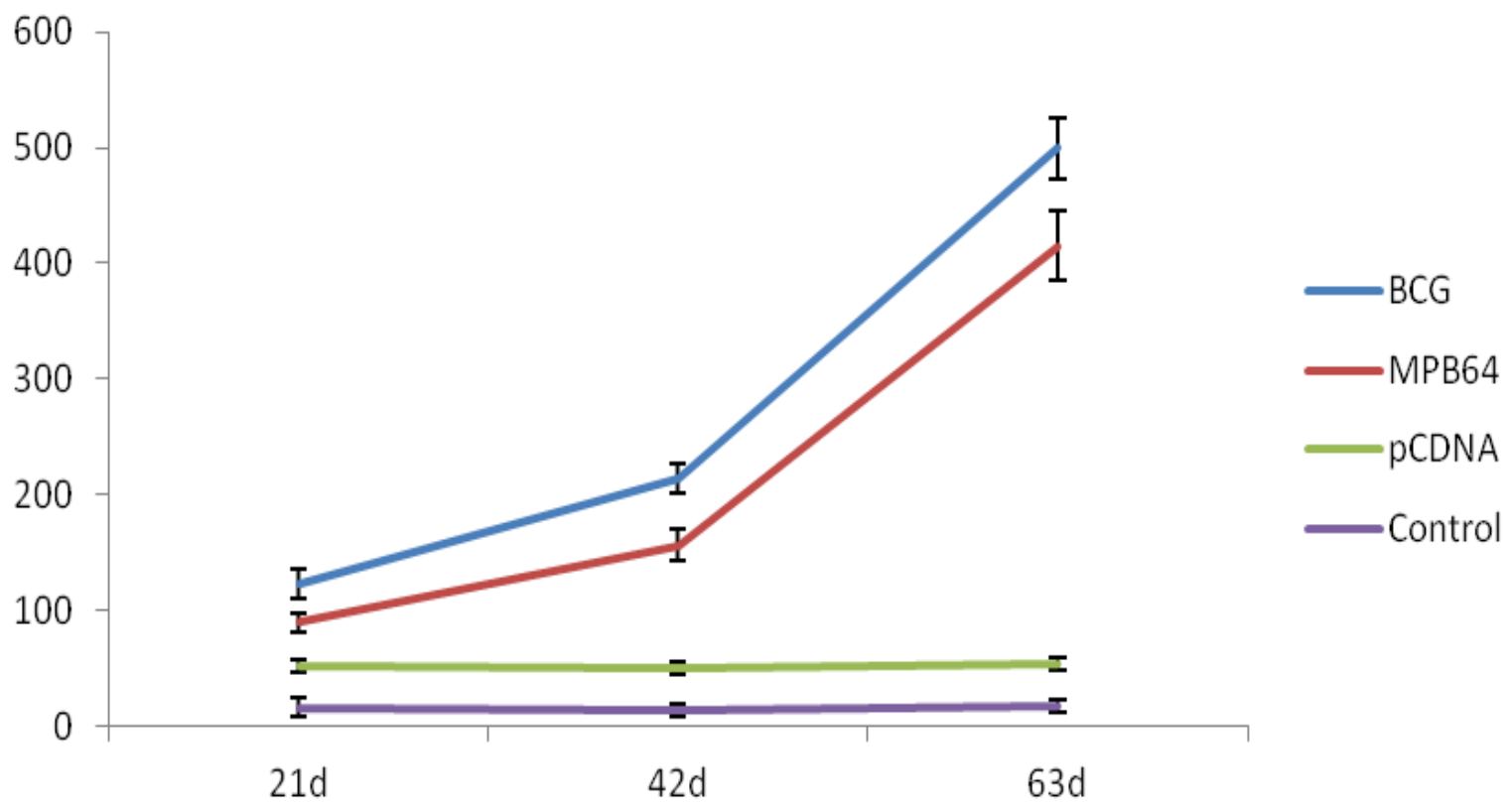

Figure 4. Antigen-specifc total IgG antibodies detected by ELISA. C57BL/6 mice $(n=20)$ were vaccinated with different vaccine candidates.

response (Dunham et al., 2006). Vector design for maximal protein expression is essential and important. This is the reason we focus on the new DNA vaccination discovery.

Antibody titer measurement is a common way to test
The quantity of antibody in human body, which has produced that recognizes a particular epitope, expressed as the greatest dilution that still gives a positive result (Decker et al., 2006). ELISA is a common used method of determining antibody titers. Our research found that 


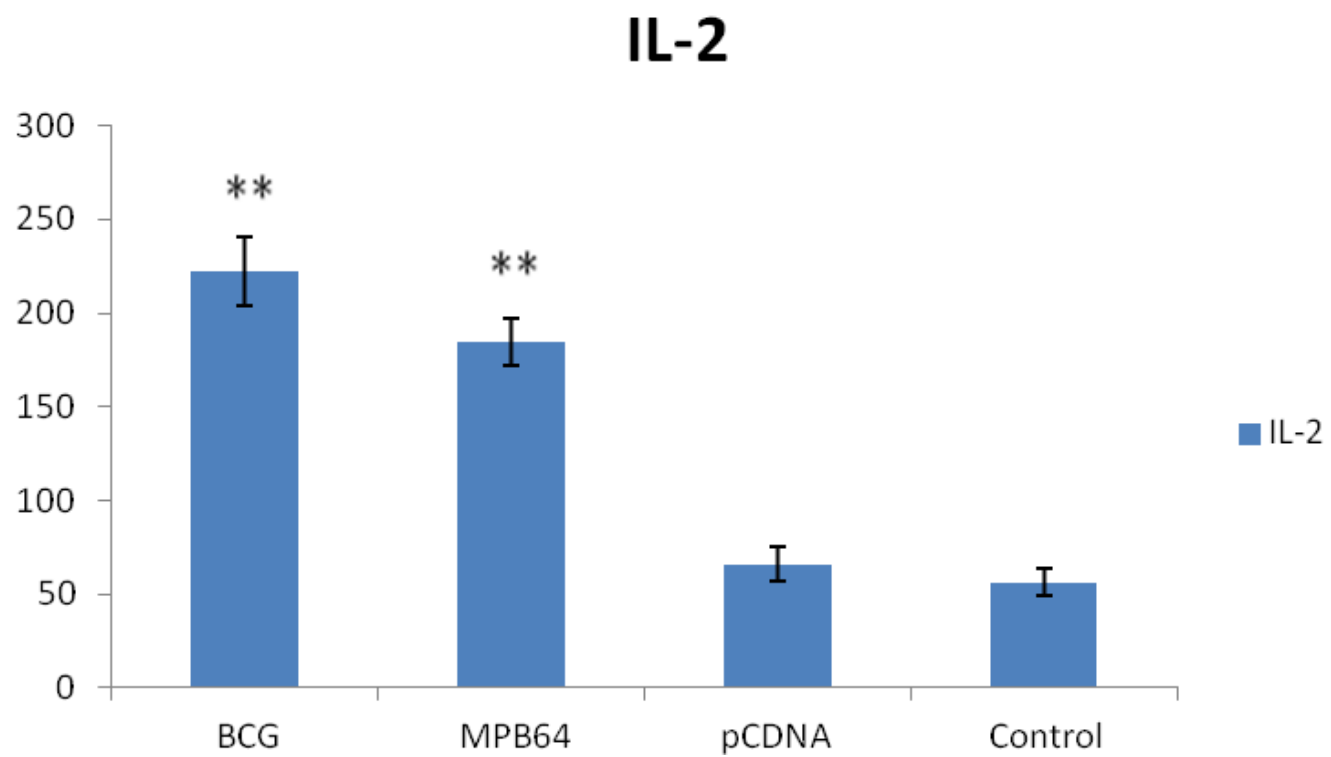

Figure 5. Antigen-specific IL-2 production of serum from mice. C57BL/6 mice $(n=20)$ were vaccinated with different vaccines. Serum was prepared 2 weeks after the last immunization. IL-2 was measured in triplicate using ELISA. The results are expressed as Mean $(\mathrm{pg} / \mathrm{ml}) \pm S D$ of ten mice in each group. Data with significant difference when $p<0.01$.

the MPB expressed and purified by the recombination expressing plasmid pCDNA-MPB64 could be enough to produce an effective immunological response. Immunogens could be targeted to various cellular compartments in order to improve antibodies or cytotoxic T-cell responses. In response to an antigen, cellmediated immunity involves the activation of natural killer cells (NK), macrophages, antigen-specific cytotoxic Tlymphocytes, and cytokines, instead of antibodies. The immune system was separated into humoral immunity and cellular immunity, for which the protective function of immunization was associated with cells (Harris et al., 2009; Lutz et al., 2009). Cell-mediated immunity is directed primarily at microbes survive in phagocytes and microbes that infect non-phagocytic cells. It is most effective in removing virus-infected cells, but also participates in defending against fungi, protozoans, intracellular bacteria, and cancers. It also plays a major role in transplant rejection in human body (Cohen et al., 2008).

The RD1 genetic region is present in the genomes of MTB and Mycobacterium bovis, but absent from all strains of $M$. bovis BCG, as well as most non-tuberculous Mycobacteria (NTM) (Pym et al., 2003). The MPB64 gene, encoded by RD1, has been investigated extensively, and has been shown to have great potential in the specific in vitro diagnosis of MTB infection in human beings (Maue et al., 2007; Kaufmann et al., 2011). Previous studies showed that RDs encode some important immuno-dominant antigens of $M$. tuberculosis and RD1 could enhance the protective efficacy of BCG in the forms of recombinant BCG or subunit vaccine.
MPB64 had been considered important immunodominant antigens encoded by RD1and RD2 of $M$. tuberculosis, respectively (Maue et al., 2007). Recent study demonstrated that DNA vaccine expressing the gene of MPB64 could strength protection efficacy, which was complied with our results (Kaufmann et al., 2011).

In conclusion, our results clearly demonstrated the vaccination of $\mathrm{C} 57 \mathrm{BL} / 6$ mice with new DNA vaccine pCDNA-MPB64 resulted in significant protection against challenge with virulent $M$. tuberculosis H37Rv when compared with the control group, which indicates that MPB64 may be an efficient booster vaccine against TB with a strong ability to enhance prior BCG immunity. These results suggest that MPB64 is safe and effective vaccine against TB.

\section{REFERENCES}

Amaral L, Viveiros M, Molnar J (2011). Effective therapy with the neuroleptic thioridazine as an adjunct to second line of defence drugs, and the potential that thioridazine offers for new patents that cover a variety of "new uses". Recent Pat. Antiinfect. Drug. Discov., 6(2): 8487.

Cohen BA, Oger J, Gagnon A (2008). The implications of immunogenicity for protein-based multiple sclerosis therapies. J. Neurol. Sci., 15; 275(1-2): 7-17.

Decker WK, Safdar A (2010). Dendritic cell vaccines for the immunocompromised patient: Prevention of influenza virus infection. Expert. Rev. Vaccines, 9(7): 721-730.

Dunham SP, Bruce J, Klein D (2006). Prime-boost vaccination using DNA and whole inactivated virus vaccines provides limited protection against virulent feline immunodeficiency virus. Vaccine, 24(49-50): 7095-7108

Frantz FG, Ito T, Cavassani KA (2011). Therapeutic DNA vaccine reduces schistosoma mansoni-induced tissue damage through 
cytokine balance and decreased migration of myofibroblasts. Am. J. Pathol., 179(1): 223-229.

Hanif SN, Al-Attiyah R, Mustafa AS (2010). DNA vaccine constructs expressing Mycobacterium tuberculosis-specific genes induce immune responses. Scand. J. Immunol., 72(5): 408-415.

Harris VK, Sadiq SA (2009). Disease biomarkers in multiple sclerosis: Potential for use in therapeutic decision making. Mol. Diagn. Ther., 13(4): 225-244.

Hawkridge T, Mahomed $\mathrm{H}$ (2011). Prospects for a new, safer and more effective TB vaccine. Paediatr. Respir. Rev., 12(1): 46-51.

Kaufmann SH (2011). Fact and fiction in tuberculosis vaccine research: 10 years later. Lancet. Infect. Dis., 11(8): 633-640.

Kernodle DS (2010). Decrease in the effectiveness of Bacille CalmetteGuérin vaccine against pulmonary tuberculosis: A consequence of increased immune suppression by microbial antioxidants, not overattenuation. Clin. Infect. Dis., 15; 51(2): 177-184.

Liang $\mathrm{Y}, \mathrm{Wu} \mathrm{X}$, Zhang $\mathrm{J}$ (2011). Treatment of multi-drug-resistant tuberculosis in mice with DNA vaccines alone or in combination with chemotherapeutic drugs. Scand. J. Immunol., 74(1): 42-46.

Lutz HU, Binder CJ, Kaveri S (2009). Naturally occurring autoantibodies in homeostasis and disease. Trends. Immunol., 30(1): 4351.

Martín MC, Gicquel B (2011). New tuberculosis vaccines. Enferm. Infect. Microbiol. Clin., Suppl 1: 57-62.
Maue AC, Waters WR, Palmer MV (2007). An ESAT-6:MPB64 DNA vaccine administered in conjunction with Mycobacterium bovis BCG confers protection to cattle challenged with virulent $M$. bovis. Vaccine, 25: 4735-4746.

Mkrtichyan M, Ghochikyan A, Movsesyan N (2008). Immunostimulant adjuvant patch enhances humoral and cellular immune responses to DNA immunization. DNA. Cell. Biol., 27(1): 19-24.

Okada M, Kita Y, Nakajima T (2011). Novel therapeutic vaccine: granulysin and new DNA vaccine against Tuberculosis. Hum. Vaccin., 7(Suppl): 60-67.

Pym AS, Brodin P, Majlessi L (2003). Recombinant BCG exporting MPB64 confers enhanced protection against tuberculosis. Nat. Med., 9: 533-539.

Rouanet C, Locht C (2010). Boosting BCG to protect against TB. Expert. Rev. Respir. Med., 4(3): 339-348.

Sala C, Hartkoorn RC (2010). Tuberculosis drugs: new candidates and how to find more. Future Microbiol., 6(6): 617-633.

Sarkar S, Suresh MR (2011). An overview of tuberculosis chemotherapy - a literature review. J. Pharm. Pharm. Sci., 14(2): 148-161. 